\title{
Can physical activity modulate pancreatic cancer risk? A systematic review and meta-analysis
}

\author{
M. A. O'Rorke, M. M. Cantwell, C. R. Cardwell, H. G. Mullholland and L. J. Murray \\ Cancer Epidemiology \& Prevention Research Group, Centre for Public Health, Queen's University Belfast, \\ Belfast BT12 6BJ, UK
}

Pancreatic cancer is the thirteenth incident malignancy in Europe but conversely is the fifth most common cause of cancer death ${ }^{(1)}$. Its aggressive growth, early metastases and late detection mean that 5-year survival is a formidable 5\% in Europe with approximately $75 \%$ of patients dying within 12 months of their initial diagnosis ${ }^{(2)}$. Despite this dismal prognosis the aetiology of pancreatic cancer remains poorly understood. Numerous epidemiological studies have examined the association between physical activity and pancreatic cancer; however, findings remain equivocal ${ }^{(3,4)}$. Physical activity improves insulin sensitivity ${ }^{(5)}$ mediating glucose intolerance and therefore could attenuate risk. A systematic review was undertaken to examine associations between physical activity and pancreatic cancer.

Six electronic databases were searched from their inception until February 2009 (PubMed, MEDLINE, EMBASE, Cochrane Library, Cumulative Index of Nursing and Allied Health Library and Science Citation Index) seeking observational studies examining any physical activity measure with pancreatic cancer incidence or mortality as an outcome. A random effects model was used to pool individual effect estimates evaluating highest $v$. lowest categories of activity.

Twenty-five studies met the criteria for inclusion. Significant reductions in pancreatic cancer risk were seen with higher levels of total (five prospective studies; relative risk (RR) 0.72 (95\% CI 0.52, 0.99)) and occupational activity (four prospective studies; RR 0.75 (95\% CI $0.59,0.96)$ ). Non-significant inverse associations between risk and recreational and transport physical activity were found. When examining exercise intensity, moderate activity was more protective (RR 0.79 (95\% CI 0.52, 1.20)) than vigorous activity (RR 0.97 (95\% CI $0.85,1.11)$ ), but results were not significant.

Significant reductions in pancreatic cancer risk were found, in the range $25-30 \%$, with higher levels of total and occupational activity. The pooled estimates showed non-significant reduced risks for pancreatic cancer with increasing recreational and transport activity in individuals reporting either walking or cycling. When examining studies of pancreatic cancer and exercise intensity neither low-intensity nor vigorousintensity exercise showed significant associations; activity of moderate intensity was slightly more protective, although this association did not reach significance.

1. Ferlay J, Autier P, Bonioil M et al. (2007) Ann Oncol 18, 581-592.

2. Michaud DS (2004) Minerva Chir 59, 99-111.

3. World Cancer Research Fund/American Institute for Cancer Research (2007) Food, Nutrition, Physical Activity, and the Prevention of Cancer: A Global Perspective. Washington, DC: AICR.

4. Pan SY \& DesMeules M (2009) Methods Mol Biol 472, 191-215.

5. Goodpaster BH, Katsiarask A \& Kelley DE (2003) Diabetes 52, 2191-2197. 